\title{
Los seguros de salud mexicanos: cobertura universal incierta
}

\author{
M exican health insurance: uncertain universal coverage
}

Asa Cristina Laurell ${ }^{1}$

${ }^{1}$ Centro deAnálisisy Estudios sobre Seguridad Social. Callejón de Chilpa 23-9, Barrio de la Concepción, Coyoacán. CP 04000 Distrito Federal M éxico.

laurel19998@gmail.com
Abstract The Mexican health system is comprised of the Department of $\mathrm{H}$ ealth, state labor social security and the private sector. It is undergoing a reform process initiated in 1995 to achieve universal coverage and separate the regulation, financing and service functions; a reform that after fifteen years is incomplete and problematic. The scope of this paper is to assess the problems that underlie the successive reforms. Special emphasis is given to the last reform stage with the introduction of the "Insurance of the People" aimed at the population without labor social se curity. In the analysis, health reform is seen as part of the Reform of the State in the context of neoliberal reorganization of society. Unlike other Latin American countries, this process did not include a new Constitution. The study is based on official documents and a systematic review of the process of the implementation of the System of Social $\mathrm{H}$ ealth Protection and its impact on coverage and access to health services. The analysis concludes that it is unlikely that universal population coverage will be accomplished much less universal access to services. H owever, reforms are leading to the commodification of the health system even in the context of a weak private sector. Key words Health insurance, Commodification, $\mathrm{H}$ ealthcare reform, $\mathrm{H}$ ealth policy, M exico
Resumen El sistema de salud mexicano se componedela Secretaría de Salud (rectora del sector y prestador de algunos servicios), la seguridad social laboral pública, y el sector privado. Transita por un proceso de reforma iniciado en 1995 para universalizar la cobertura y separar las funciones regulación-financiamiento-prestación de servicios; reforma que después de quince años sigue inacabada y problemática. Este texto analiza crítica- y propositivamente la problemática surgida a raíz de las sucesivas reformas. El énfasis se pone en su última etapa con la introducción del "Seguro Popular" para la población sin seguridad social laboral. El análisis concibe la reforma de salud como parte de la Reforma del Estado en el marco de la reorganización neoliberal de la sociedad. A diferencia de otros países latinoamericanos este proceso no pasó por una nueva Constitución. EI análisis se basa en documentos oficiales y un seguimiento sistemático de la instrumentación del Sistema deProtección Social en Salud y su impacto sobre la cobertura y acceso a los servicios. Se concluye que es improbable que se universalice la cobertura poblacional y menos el acceso a los servicios. Empero las reformas están forzando la mercantilización del sistema aún en presencia de un sector privado débil.

Palabras clave Seguro de salud, M ercantilización, Reforma de la atención de salud, Política de salud, M éxico 


\section{Introducción}

El sistema de salud mexicano tiene dos características básicas: su alto grado de segmentación y el importante peso de las instituciones públicas en la prestación de servicios. La segmentación del sistema se deriva deuna política social que concibió la construcción del bienestar social a partir dela incorporación dela población crecientemente asalariada a la seguridad social, iniciada en el periodo de posguerra. Este modelo fue exitoso hasta los años ochenta ya que la cobertura de las dos grandes instituciones públicas de seguridad social -el Instituto M exicano de Seguridad Social (IM SS) para los trabajadores del sector privado y el Instituto de Seguridad y Servicios Sociales de los Trabajadores del Estado (ISSSTE) para los del sector público- creció rápidamente ${ }^{1}$. Sin embargo, a partir del "ajuste estructural" iniciado en 1983, se frenó la disminución de la población objetivo de la Secretaria (M inisterio) de Salud (SSA) - la no asegurada o "abierta" - por el crecimiento del desempleo y el empleo informal².

El primer intento de unificar las instituciones públicas de salud se hizo a finales de los ochenta. Al fracasar la unificación se inició un proceso de descentralización de la SSA en 1989; proceso que sefrenó por problemas institucionales con el virtual colapso dela atención médica quecausó protestas populares importantes 3 . El actual proceso dereforma seinició con el IM SS en 1995, pasó por laconstitución del Sistemanacional deProtección Social en Salud (SN PSS) en 2003 y culminó con la reforma del ISSSTE en 2007. El esquema básico de esta prolongada e inacabada reforma es la separación entrelas funciones de regulación, administración del financiamiento y la prestación de servicios quesignifica la mercantilización y abrea la privatización. Su argumento retórico principal es universalizar la cobertura de salud.

El presentetexto tiene como propósito analizar la problemática del sistema de salud surgida a raíz de estas reformas parciales sucesivas. Se pondrá un énfasis particular en el Seguro Popular (SP) para la población carente de seguridad social laboral quees el eje sobre el cual el gobierno federal promete universalizar la cobertura en 2011. Revela además con claridad las características del modelo que se intenta implantar.

En el análisis se concibe al proceso de reforma de salud como parte de la Reforma del Estado en el marco de la reorganización neoliberal dela sociedad. A diferencia de otros países latinoamericanos este proceso no está acompañado por la democratización o una nueva Constitución en
M éxico. El examen se realiza con base en los documentos oficialesy en el seguimiento sistemático del proceso de instrumentación del SNPSS y el Seguro Popular en sus aspectos financiero, institucional, organizativo y su impacto sobre la cobertura y acceso a los servicios de salud. En el análisis se contrastan además el discurso oficial con los hechos por la constante tensión entre lo quese dicey lo queen la realidad está ocurriendo.

\section{Las Reformas de los Institutos de Seguridad Social}

Los seguros médicos o de salud incluidos en la seguridad social laboral cubren alrededor del $45 \%$ de la población que son los trabajadores formales y sus familias. El IM SS es el instituto más importante con el $76 \%$ de los derechohabientes, seguido por el ISSSTE con otro $15 \%$ mientras que el 9\% restante se reparte entre los institutos de seguridad social de la paraestatal PEM EX, de las fuerzas armadas y de al gunos estado ${ }^{4}$. Los institutos difieren en el ámbito de las pensiones y otros beneficios mientras que las prestaciones de salud son iguales y cubren prácticamente todos los servicios de salud incluyendo los medicamentos.

Los institutos de seguridad social laboral son empresas paraestatales desconcentradas, sujetas al control presupuestal y al poder ejecutivo federal. Todos prestan una proporción muy alta de sus servicios médicos en instalaciones propias y con personal asalariado. Disponen de los recursos materiales y humanos para la salud más importantes del país. En 2007 concentraban cerca de 42 mil camas censables en 465 hospitales y empleaban a 78 mil médicos y 118 mil enfermeras $^{5}$. El IM SS subroga menos del $5 \%$ de sus servicios con el sector privado mientras queel ISSSTE a penas alcanza un $10 \%$.

La reforma del IMSS se dio como parte del tercer "ajuste estructural" después de la profunda crisisfinanciera en 1994. En esta coyuntura el Banco Mundial proporcionó a M éxico un préstamo de estabilización que tenía entre sus "condicionalidades" esta reforma. Pese a que se preveía la introducción de múltiples administradores de fondosy prestadores de serviciostanto públicos como privado $s^{6,7}$, este planteamiento no ha prosperado. Una razón es la notable dificultad dedesmontar una institución degrande tamaño y complejidad sin provocar serios tropiezos en la prestación de servicios. Otra es la pertinaz resistencia de los trabajadores del IM SS a su desmontaje. Actualmente se desarrollan ensayos de autonomía de 
gestión en los hospitales de alta especialidad pero aparentemente sin mucho éxito.

Sin embargo la reforma ha contribuido a debilitar el seguro de salud del IM SS. Estableció la separación entre los fondos de los cinco ramos del seguro social como condición necesaria para introducir la capitalización individual con administración privada de los fondos de pensiones. Esto imposibilitó el uso cruzado entrelos distintos fondos que había sido una fuente importante de financiamiento del seguro de salud. Otro cambio de consecuencias adversas fue la sustitución de la aportación patronal proporcional al salario por una prima única por trabajador. Este esquema regresivo disminuyó la aportación patronal a partir de los 3 salarios mínimos y éste pasó del $8.75 \%$ a un rango deentre $7.5 \%$ y $2.62 \%$ sobre el salario base 2 . Esto impidió que se resolviera el subfinanciamiento del seguro de salud a pesar de que la aportación estatal fiscal se incrementara de un 5 a un 30\% del financiamiento total. Además la ley fijó la contribución para los servicios médicos de los pensionados en el 1.5\% sobre la masa salarial que ha mostrado ser insuficiente y la causa principal del desfinanciamiento de los servicios de salud8.

La estrechez financiera, los bajos salarios y el lento crecimiento del empleo han conducido a un prolongado deterioro del IMSS, antaño la mejor institución pública de salud en América Latina. Semanifiesta en la falta demedicamentos einsumos; problemas demantenimiento de equipo e instalaciones; retrazo en la edificación de nueva infraestructura y en sustitución de equipo e; inestabilidad contractual del nuevo personal. No obstante el I M SS sigue siendo un prestador deservicios muy importante. Proporcionael $40 \%$ delas consultas del sector público, atiendeel $58 \%$ de las urgencias y le corresponde el $68 \%$ de los egresos hospitalarios; datos todos más al tos que la proporción de la población a su cargo ${ }^{9}$.

La reforma del ISSSTE se dio apenas en 2007, diez años después dela del IM SS. Sepospuso por el papel de los trabajadores del Estado como sostén político del gobierno en turno. La propuesta inicial de reforma fue presentada por el máximo dirigente sindical y apoyada por la poderosa dirigente del sindicato de maestros con un millón de trabajadores. La nueva ley se aprobó atropeIladamente en el Congreso a pesar deque cientos demiles detrabajadores se ampararon en su contra. Significativamente la administración de los nuevos fondos de pensiones con cobros de altas comisiones quedó en manos de estos dirigentes. Adicionalmente el yerno de la dirigente magiste- rial fue nombrado viceministro de educación y un aliado suyo director del ISSSTE.

La nueva ley ${ }^{10}$ es casi una calca de la ley de IMSS a pesar del reconocimiento público de sus fracasos ${ }^{11}$. Su seguro de salud abarca genéricamente la atención médica preventiva, curativa y de maternidad y rehabilitación física y mental (art. 27). Se establece explícitamente la separación entrela administración del fondo de salud y la prestación de los servicios médicos (art. 2931). Queda a cargo de una instancia administradora la compra de los servicios de salud a las unidades médicas del instituto 0 a otros prestadores incluyendo a los privados. 0 sea, se introducen mecanismos de competencia con mercados interno y externo. La instancia administradora también debe garantizar que el techo presupuestal no sea rebasado; tarea que implica fijar el precio de los servicios, determinar cuales prestar gratuitamente $y / 0$ eventualmente introducir copagos y seguros complementarios para cubrir los servicios excluidos. Así el seguro de salud tieneuna plena lógica mercantil y prefigura una disminución de los servicios autorizados.

El esquema de financiamiento del seguro de salud del ISSSTE difiere de él del IMSS ya que mantiene la aportación patronal proporcional al salario base con un techo de diez salarios mínimos y el trabajador paga una prima varias veces mayor que la del IMSS (Tabla 1). La cotización para el seguro de salud de los pensionados es el mismo que en el I M SS a pesar de su insuficiencia. Aunqueel nivel salarial promedio del sector público sea más alto que en el sector privado y nunca por debajo de un salario mínimo, es incierta la sustentabilidad financiera del seguro. De allí la predicción de una próxima restricción de servicios.

Aunqueel ISSSTE tenga múltiples problemas en la prestación de servicios también posee importantes recursos para esta tarea. Según los últimos datos disponibles la prestación de servicios por derechohabienteseacerca a los del I M SS. Recibió además al aprobarse la reforma recursos públicos extraordinarios de unos US\$ 640 millones para ampliar su infraestructura. Sigue siendo un coto de poder del sindicato y hay indicios fuertes de una sistemática corrupción institucional y tráfico de influencias.

\section{El Seguro Popular ante la seguridad social obligatoria}

El eje de la fase actual de la reforma es el Sistema Nacional deProtección Social en Salud (SN PSS) 
Tabla 1. Comparación de la cotización en pesos mexicanos al Seguro Popular, Instituto M exicano de Seguridad Social (IMSS) e Instituto de Seguridad y Servicios Sociales de los Trabajadores del Estado (ISSSTE) por decíl de ingreso*, 2008.

\begin{tabular}{lrrrrr}
\hline $\begin{array}{l}\text { Decíl de } \\
\text { ingreso }\end{array}$ & $\begin{array}{c}\text { Ingreso } \\
\text { promedio } \\
\text { anual** }\end{array}$ & $\begin{array}{c}\text { Número } \\
\text { de SM }\end{array}$ & $\begin{array}{c}\text { Prima } \\
\text { Seguro } \\
\text { Popular }\end{array}$ & $\begin{array}{c}\text { Prima del } \\
\text { trabajador al } \\
\text { I M SS }\end{array}$ & $\begin{array}{c}\text { Prima del } \\
\text { trabajador al } \\
\text { ISSSTE }\end{array}$ \\
\hline I & 24,464 & 0.32 & 0 & No paga & No aplica \\
II & 42,748 & 0.56 & 0 & N o paga & No aplica \\
III & 57,572 & 0.75 & 714 & N o paga & No aplica \\
IV & 71,900 & 0.94 & 1,400 & N o paga & No aplica \\
V & 87,804 & 1.14 & 2,075 & No paga & 2,415 \\
VI & 108,032 & 1.41 & 2,834 & No paga & 2,971 \\
VII & 134,912 & 1.76 & 3,648 & N o paga & 3,710 \\
VIII & 171,400 & 2.23 & 5,650 & N o paga & 4,714 \\
IX & 236,728 & 3.08 & 7,519 & 947 & 5,279 \\
X & 532,192 & 6.93 & 11,379 & 1,920 & 5,279
\end{tabular}

Fuente: ${ }^{*}$ Encuesta de Ingreso y Gasto de los $\operatorname{Hogares}^{12} ;{ }^{* *} \mathrm{CNPSS}^{13}$ (p. 12). La prima está calculada sobreel ingreso promedio anual por decíl de ingreso.

y el Seguro Popular para la población sin seguridad social laboral. La legislación correspondientefue aprobada en 2003 con el voto en contra de los diputados de izquierda. La oferta del SN PSS es que se logrará la "cobertura universal" en 2010 o 2011. Hay que señalar que es la segunda vez que el gobierno federal promete la cobertura universal y ha lanzado varios programas de extensión de cobertura durante las últimas décadas. El más importantefue un nuevo subsistema público, administrado por el I M SS y financiado con recursos fiscales, para la población rural e indígena. Logró la cobertura de unas 10 millones depersonas entre 1980 y 1982 a través dela construcción de una extensa infraestructura de salud - 42 hospitales con 25-30 camas y equipados con rayos- $X$ y laboratorio así como 4 mil clínicas-y la contratación de personal calificado ${ }^{14}$.

En 1995 se adoptó una política de "cobertura universal" como parte del Ilamado "Programa de Reforma del Sector Salud". Sin embargo, esta cobertura universal era sólo de un paquete de "Servicios Esenciales" de 16 intervenciones ${ }^{15}$ alineada con el combate a la pobreza.

La constitución del SN PSS significó el abandono del fortalecimiento eintegración delos servicios públicos de salud para extender la cobertura medianteel aseguramiento siguiendo el planteamiento dominante. La denominación "cobertura" se refiere a la cobertura "poblacional" y no a la "de servicios de salud".
Para entender el al cance del Seguro Popular (SP) es conveniente compararlo con el seguro de salud de los institutos de seguridad social cuyos aspectos sobresalientes se resumen en el Cuadro 1 con el IM SS como ejemplo. Las diferencias están sancionadas por dosmarcos legalesdistinto, parcialmente contradictorios o sobrepuestos. Deesta manera, la protección dela salud está reconocida en el artículo 4 constitucional como un derecho, pero carece de un sujeto obligado a garantizarla, y reglamentada en la Ley General de Salud. La seguridad social emana del artículo 123 constitucional sobre los derechos del trabajo, con el Estado yel patrón como sujetos obligados cuyos deberes están reglamentados en las leyes de Seguridad Social (Cuadro 1).

La primera discrepancia entre ambos regímenes es que el SP es voluntario mientras que el seguro médico laboral es obligatorio. La persona o la familia debe, en principio, solicitar la inscripción y pagar una prima al SP o a alguna de sus modalidades, el Seguro M édico para una Nueva Generación (SM NG) para los nacidos durante el actual gobierno o el Programa de Embarazo Saludable (PES). EI SM N G tiene inocultables tintes políticos y discrimina contra los niños nacidos antes del 2007. En contrastela inscripción del trabajador a la seguridad social laboral esobligación del patrón y su incumplimiento sancionable. Los jubilados y pensionados por los institutos deseguro social y sus familias mantienen la cobertura del seguro médico. 
Cuadro 1. Características del Seguro Popular y de la Seguridad Social Laboral.

\begin{tabular}{|c|c|c|}
\hline Concepto & Seguro Popular & $\begin{array}{c}\text { Seguridad Social Laboral } \\
\text { Instituto M exicano de Seguridad } \\
\text { Social (IMSS) }\end{array}$ \\
\hline Criterios de inclusión & $\begin{array}{l}\text { Voluntario. } \\
\text { No tener seguridad social laboral. } \\
\text { Solicitar inscripción. } \\
\text { Pagar cuota (prima) familiar. } \\
\text { Renovar anualmente inscripción y pago. } \\
\text { Haber nacido después del último cambio de } \\
\text { gobierno federal (1/12/2006) en una familia } \\
\text { sin seguridad social laboral. } \\
\text { Estar embarazada sin seguridad social } \\
\text { laboral. }\end{array}$ & $\begin{array}{l}\text { Obligatorio. } \\
\text { Ser asalariado o familiar directo } \\
\text { de asalariados. } \\
\text { Ser pensionado del IMSS. }\end{array}$ \\
\hline $\begin{array}{l}\text { Criterios de } \\
\text { exclusión }\end{array}$ & $\begin{array}{l}\text { No reinscribirse o no pagar la cuota familiar. } \\
\text { Adquirir seguridad social. } \\
\text { Cláusulas de exclusión definitiva. }\end{array}$ & $\begin{array}{l}\text { Perder el empleo antes de } 60 \\
\text { años o con cotización menor de } \\
750 \text { semanas. } \\
\text { Existe continuidad voluntaria } \\
\text { del seguro. }\end{array}$ \\
\hline Beneficiarios & Titular y su familia. & Asegurado y sus familiares directos. \\
\hline Cupo de afiliados & $\begin{array}{l}\text { 14.3\% de las familias susceptibles al año } \\
\text { (cobertura universal en } 2010 \text { o 2011). }\end{array}$ & $\begin{array}{l}\text { Al ser obligatorio no tiene cupo } \\
\text { de asegurados. }\end{array}$ \\
\hline Financiamiento & $\begin{array}{l}\text { "Tripartita": Estado federal- entidad } \\
\text { federativa-familia. Prima familiar según el } \\
\text { ingreso. } \\
\text { La ley prevé cuota moderadora. }\end{array}$ & $\begin{array}{l}\text { Tripartita: Patrón-trabajador- } \\
\text { Estado. } \\
\text { N o existe co-pago u otras cuotas } \\
\text { aparte de la contribución sobre } \\
\text { el salario. }\end{array}$ \\
\hline $\begin{array}{l}\text { Beneficios } \\
\text { garantizados }\end{array}$ & $\begin{array}{l}\text { Paquete de servicios y medicamentos } \\
\text { especificados en el Catálogo Universal de } \\
\text { Servicios de Salud (CAUSES) y cuatro de } \\
\text { Gastos Catastróficos y los incluidos en el } \\
\text { Seguro M édico para una N ueva Generación } \\
\text { (SM N G). } \\
\text { Todo los demás servicios se pagan. }\end{array}$ & $\begin{array}{l}\text { Servicios de Salud } \\
\text { Asistencia médico quirúrgica y } \\
\text { obstétrica, farmacéutica y } \\
\text { hospitalaria necesaria. } \\
\text { Servicios de medicina preventiva } \\
\text { y rehabilitación. } \\
\text { Prestaciones en dinero } \\
\text { Subsidios enfermedad y por } \\
\text { maternidad. }\end{array}$ \\
\hline $\begin{array}{l}\text { Organización } \\
\text { institucional }\end{array}$ & $\begin{array}{l}\text { Separación de funciones y estructuras: } \\
\text { SSA regula el sistema; } \\
\text { CNPSS y REPSS administran el } \\
\text { financiamiento y compran servicios; } \\
\text { SSA y SES prestan los servicios según la } \\
\text { LGS. } \\
\text { EI reglamento permite prestación de } \\
\text { servicios por parte de los institutos de } \\
\text { seguridad social y el sector privado. }\end{array}$ & $\begin{array}{l}\text { Asamblea tripartita máximo } \\
\text { órgano de decisión. } \\
\text { Consejo Técnico tripartita. } \\
\text { Integrado con regulación, } \\
\text { administración del } \\
\text { financiamiento y prestación de } \\
\text { servicios. } \\
\text { Intentos de separación de } \\
\text { funciones, actualmente sólo } \\
\text { autonomía de gestión hospitales } \\
\text { tercer nivel y reversión de cuotas. }\end{array}$ \\
\hline Prestador del servicio & $\begin{array}{l}\text { H ospitales autónomos y centros de salud de } \\
\text { los ses según la LGS. EI reglamento permite } \\
\text { prestación interinstitucional y con } \\
\text { prestadores privados vía convenios o } \\
\text { acuerdos de gestión. }\end{array}$ & $\begin{array}{l}\text { Directa en servicios del IMSS. } \\
\text { Indirecta mediante convenio } \\
\text { con instituciones prestadoras de } \\
\text { servicios públicos o privados. } \\
\text { Servicios propios del patrón } \\
\text { (subrogado) con reversión de } \\
\text { cuotas. }\end{array}$ \\
\hline
\end{tabular}

Fuente: Elaboración propia con base en la Ley General de Salud ${ }^{11}$ y su Reglamento y la Ley del Seguro Social ${ }^{16}$. SES = Servicios Estatales de Salud. 
Los criterios deexclusión del SP son no pagar la cuota familiar o no reinscribirse 0 adquirir seguridad social obligatoria. Existen además cláusulas de exclusión definitiva en caso de mal uso del seguro lo quees anticonstitucional al tratarse de un derecho. La exclusión del seguro médico laboral ocurre por la terminación de la relación laboral antes de la edad de jubilación o en el caso deno haber cotizado 750 semanas, condición que se introdujo con la reforma.

Al iniciarse el SP se estableció un cupo anual máximo del $14.3 \%$ de la población objetivo, o sea, la cobertura universal tardaría siete años o hasta 2010. Se ha sostenido ${ }^{17}$ que este límite se negoció con el ministerio de finanzas a cambio de que el incremento presupuestal correspondientequedara en la ley; afirmación que no tiene fundamento alguno ${ }^{18}$. La afiliación selectiva al SP ha generado un nuevo segmento en el sistema de salud. Los afiliados gozan de beneficios distintos al resto de la población que se atiende en las mismas unidades desalud y con el mismo personal. Esta discriminación se manifiesta, por ejemplo, en que los afiliados tienen preferencia a todo tipo de atención; reciben medicamentosgratuitos (si existen); cuentan con un gestor desusintereses en los hospitales; etcétera. Si esta nueva segmentación fuera temporal se podría aceptar como un problema de transición pero como se verá es poco probable que se logre la cobertura universal.

Se presenta el esquema definanciamiento del SP como equivalente al de la seguridad social ${ }^{19}$. Sería "tripartita" donde las tres partes son el Estado central, los estados federados y la familia. Seequipara efectivamentela aportación del Estado central al SP con la de los seguros obligatoria de la seguridad social ya que proporciona una "cuota social" igual 15\% del salario mínimo (SM ) a todos los esquemas. El SP recibe además una "cuota solidaria federal" de $22.5 \%$ del SM. Sin embargo ésta se descuenta de la transferencia presupuestal federal regular a las Secretarías Estatales de Salud (SES) y no siempre incrementa sus recursos sino los reorienta al SP. Esto significa queen cuanto avancela cobertura se dará toda la transferencia federal vía el SP, o sea, se transitará del subsidio a la oferta (a los servicios) al subsidio a la demanda (el recurso sigueal "consumidor"). Este es principio básico y condición de la mercantilización del sistema de salud.

Los estados deben aportar una cuota fija e igual por cada familia afiliada. Es un arreglo inequitativo ya que los estados más pobres y con menos ingresos fiscales tienen la menor cobertura de seguridad social y, por tanto, proporcional- mente más familias por subsidiar. Por ejemplo, la cobertura de seguridad social laboral en Chiapas, el estado más pobre de M éxico, es del 18\% mientras que en Nuevo León, el estado más rico, la cobertura es de $69 \%$. Esta inequidad pudiera convertirseen un obstáculo a la universalización dela cobertura por falta de recursos estatales y porque afecta otras inversiones sociales básicas. Sin embargo, hay indicios de que no se han incrementado sustancialmente las aportaciones estatales en la práctica sino más bien hay una nueva contabilidad desu gasto. Por último está la aportación de la familia al SP y el trabajador a la seguridad social dondesevuelvea presentar la inequidad como se observa en el Tabla 1.

La ley exenta de pago a las familias que pertenecen a los deciles I y II pero las pertenecientes a los deciles III y IV deberían cotizar sin ganar siquiera un salario mínimo (SM). El SM NG y las mujeres del PES constituyen las excepciones y están exentas hasta el decil VIII. El SP considera que la familia debe pagar el 3-4\% de su ingreso al seguro de salud. En contraste, el trabajador empieza a cotizar el $0.4 \%$ sobre el salario base al seguro médico del IM SS a partir de los tres SM hasta un techo de 25 SM o un máximo de 1,920 pesos. Para el seguro del ISSSTE, donde el salario nunca es menor a un SM, el trabajador cotiza el $2.75 \%$ sobre su salario base hasta el techo de diez SM con una prima máxima de 5,280 pesos (Tabla 1). Adicionalmente, el SP es el único seguro publico que tiene previsto un co-pago, la "cuota moderadora". De esta manera los afiliados al SP son los que según las reglas deberían pagar más por su seguro seguidos por los del ISSSTE. Sin embargo pese a que se levanta una cédula socio-económica a la familia al afiliarla prácticamente nadie paga la prima supuestamente por pertenecer al $20 \%$ más pobre o por inscribirse en el SM NG o el PES.

Estos datos muestran que quien más debería depagar recibemenos beneficios. El seguro médico laboral cubre todas las intervenciones mientras que el SP sólo ampara el Catálogo Universal deServicios deSalud (CAUSES), cuarto enfermedades de gasto catastrófico para adultos y 18 para los menores de cinco años. Según la Comisión N acional deProtección Social en Salud (CN PSS) ${ }^{20}$ el CAUSES, con 266 "intervenciones" y los medicamentos correspondientes, cubre todos los servicios ambulatorios y el $98 \%$ de las hospitalizaciones del segundo nivel, afirmación insostenible confrontándolo con los datos de las causas de egresos hospitalarios?.

Los padecimientos de gasto catastrófico cubiertos para adultos son: cáncer mamario y cér- 
vico-uterino, tratamiento ambulatorio de VIH SIDA con antiretrovirales y cirugía de cataratas. Padecimientos muy comunes como el infarto al miocárdio, la hemorragia cerebral, los politraumatismos y la mayoría de los cánceres no están cubiertos por el CAUSES o como gasto catastrófico y deben ser pagados por los asegurados. Por otra parte es de subrayar que la inclusión/exclusión de intervenciones tiende a fracturar el proceso integrado deatención.

La cobertura médica de los niños es mayor y revela que el actual gobierno ha hecho su propio esquema de seguro que privilegia al SM N G. EI SP normal ha pasado crecientemente a considerarse un programa de combate a la pobreza antes que un seguro para universalizar el derecho a la salud; concepción queseconfirma en los documentos sometidos al Banco Mundial para conseguir prestamos para el SP21.

La organización institucional del SNPSS es distinta a la del IM SS y apunta hacia la separación de las funciones "administración defondoscompra de servicios" y prestación de servicios. La CN PSS -órgano desconcentrado de la SSA con un comisionado nombrado por el presidente- es la máxima instancia del Sistema. Esta estructura se reproduce en los estados donde la ley estableceque debiera existir un "Régimen" deProtección Social en Salud (REPSS). Depende, en rigor, del gobernador y mantiene una relación de autonomía relativa respecto a la SES. EI REPSS es ambiguo en la ley pero sedefineen el Reglamento expedido por el ejecutivo federal. Esta imprecisión fue una maniobra para hacer pasar la ley por el Congreso como también lo fue el quitar a los prestadores privados en la ley para volverlos a introducir en el Reglamento ${ }^{14}$.

Es esta estructura la que permite la separación de funciones dondelaSSA sólo regula el sistema; la CN PSS y los REPSS administran fondos y compran servicios y; los SES prestan los servicios de acuerdo con la ley. Sin embargo como ya se señaló el Reglamento de la ley autoriza quelos institutos de seguridad social y, sobre todo, los privados presten los servicios. Esto plantea una pregunta central. ¿H ay un intento real de construir las condiciones de acceso mediante el sistema público o el SP es una palanca para forzar la mercantilización del sistema público de salud?
¿Hacia el cumplimiento

de las promesas del Seguro Popular?

Empecemos por analizar la cobertura poblacional real del SP y la posibilidad de que sellegue al aseguramiento universal entre éste y los seguros de salud obligatorios. La cobertura poblacional universal en el segmentado sistema mexicano depende del SP ya quesu población objetivo conceptual y legal es la población total menos la asegurada laboralmente. Debería incorporar a todo aquel que no tiene otro seguro, con independencia desu condición social y económica.

La población objetivo fue fijada en números absolutos en 2004 sin incluir el crecimiento poblacional. Esteomisión fue parcial mente corregida en 2008 subiendo lasfamilias objetivo de 11.9 a 12.6 millones pero repitiendo el error de no proyectar el crecimiento dela población y del empleo informal. Esterecal culo añadeotra inconsistencia ya que quita arbitrariamente 1.3 millones de familias ubicadas en los deciles IX y X porque "no estarían interesadas en incorporarse" al SP ( $p$. 14) ${ }^{13}$. Esto equivale a decir, no habrá cobertura universal porque las familias no quieren.

En 2009 la Sistema de Protección Social en Salud (CNPSS) reportó una cobertura de 10.5 millones defamilias o el $83 \%$ delas susceptiblesy 31 millones de personas o el 53\% de las asegurables o el $29 \%$ de la población total; datos que resultan difíciles de conciliar. Si se comparan estos datos con los de la Encuesta Nacional de Ingreso y Gasto de los Hogares ${ }^{12}$, la cobertura resulta todavía más incierta ya quela cobertura del SP sólo sería del 71 al $73 \%$ de las familias afiliables aun antes de la crisis económica actual.

La CN PSS ha dado varias explicaciones a la discrepancia entreel número defamilias y depersonas pero ninguna es satisfactoria. Probablementehay un registro erróneo de los estados para acceder a mayores recursos económicos y una alteración de los datos por la Comisión para simular quese avanza a la cobertura universal. Esta afirmación se sustenta en varias incoherencias de la CNPSS. Informa ${ }^{20}$ queen 20098.7 millones de las familias afiliadas están encabezadas por mujeres lo que sobrepasaría en unos 2 millones el total de este tipo de familias. Los datos respecto al nivel de ingreso de las familias afiliadas son igualmente asombrosos ya que en $200910.1 \mathrm{mi}-$ Ilones de familias pertenecerían al $20 \%$ más pobre del país lo que es el $190 \%$ de los 5.3 millones de este nivel. Probablemente estas inconsistencias se deban a quese mezclan los datos del Seguro M édico para una Nueva con los del SP; hecho 
que se confirma por un número muy alto de familias unipersonales.

Por otra parte, los datos oficiales ${ }^{22}$ revelan que los recursos financieros asignados al SP son insuficientes y adicionalmente mal ejercidos. EI SP ha significado un incremento significativo en los recursos destinados a la SSA y los SES. Sin embargo hay varios problemas a los cuales no se han prestado la suficienteatención. El primero es que el presupuesto público total de salud (suma de la seguridad social y de la SSA) como porcentaje del PIB tiene un incremento exiguo de 2.6 a 2.8 entre 2003 y 2008. Si se desglosan los datos entre la seguridad social y la SSA, incluyendo el $\mathrm{SP}$, resulta que los recursos deaquella bajaron en $0.19 \%$ del PIB mientras que los últimos aumentaron en $0.39 \%$ del PIB. El incremento de recursos financieros se ha canalizado sobre todo al SNPSS y en menor medida al resto de las actividades de la SSA/SES.

La aplicación de los fondos del SP demuestra un subejercicio sistemático, aunque aparentemente no ha sido el caso. El análisis detallado descubre que parte del dinero se ha depositado en el Fideicomiso de Protección Social en Salud (FPSS) en vez deejercerlo en el fortalecimiento de las actividades de atención. El mecanismo utilizado es que se transfiereal rededor del $23 \%$ delas aportacionesfederales al FPSS en vez del $11 \%$ legal ${ }^{13}$. Este procedimiento no hubierasido del todo reprobable si estos recursos se habrían usados para incrementar los padecimientos autorizados de gasto catastrófico o para aumentar la capacidad de atención pero no se ha hecho.

EI FPSS ha acumulado año con año cientos de millones de dólares llegando a un remanente de US\$1.5 mil millones a finales de $2009^{23}$. Las reducciones presupuestales autorizadas sesuman a estos recursos ocultos y el subejercicio anual ha correspondido grosso modo al incremento presupuestal respecto al año anterior. A pesar del dinero congelado, el gobierno mexicano contrató un préstamo con el BM de US\$ 1.25 mil millones supuestamente para financiar parte de las transferencias federales al SP en $2010^{21}$.

Una tercera incongruencia en el financiamiento del SP, poco conocida, es que los recursos solicitados por la SSA y autorizados por la Cámara de Diputados son sustancialmente menores al costo calculado del CAUSES. El documento de evaluación del BID $^{24}$ revela que la SSA estimó el costo del paquete inicial de servicios con menos intervenciones en U $\$ 625$ por familiay un incremento del 10\% para nuevas intervenciones. Esto significa que el presupuesto para la CNPSS en
2009 debería de haber sido, en un cálculo muy conservador, US $\$ 6,600$ millones y no US $\$ 3,481$ millones ( $p .14)^{13}$. Esta circunstancia ya fueseñalada en la auditoría al SP de $2005^{25}$ pero no se ha corregido el presupuesto. Por el contrario, a partir de 2010 se disminuyó la transferencia a los estados por familia en un tercio con el argumento de las familias afiliadas tienen menos miembros que los calculados (p. 85) ${ }^{20}$.

También hay carencias de recursos públicos, físicos y humano, que siguen sien do muy insuficientes y actual mentela contratación de servicios privados es muy baja. A pesar de las frecuentes declaraciones oficiales sobrela ampliación deservicios e inversión en infraestructura sus propios datos demuestran lo contrario. Por ejemplo, las camas censables por 1,000 habitantes es en promedio bajo, el 0.8 , pero 10 estados tienen 0.50 menos. En cuanto a los médicos hay en promedio 1.5 por 1,000 habitantes pero cinco estados tienen uno o menos. Adicionalmente el $30 \%$ de éstos están en formación y en seis estados corresponden al $35 \%$ o más de los médicos ${ }^{26}$.

Dado que la cobertura del SP es de primer y segundo nivel es llamativo que la mayor inversión federal ha sido en hospitales de alta especialidad, sea bajo el costosísimo esquema de asociación público-privada o el tradicional. Esta inversión no ha dado resultados ya que los seis hospitales de alta especialidad construidos por los últimos dos gobiernos han tardado meses en arrancar, están crónicamente subocupados y trabajan en el mejor de los casos al $40 \%$ de su capacidad ${ }^{27}$.

La prueba más contundente de que el SP no está cumpliendo su cometido demejorar el acceso a los servicios requeridos está en sus propios datos de atención. En el Tabla 2 se comparan sus datos con los datos de servicios prestados por las principales instituciones públicas de salud. En el caso del SP se utilizó como denominador las personas afiliadas al 1 de julio de 2009.

El Tabla 2 muestra que los servicios prestados a los afiliados del SP comparan desfavorablemente con los de las otras instituciones en todos los rubros, incluso con los de la SSA. Es de subrayar que las diferencias no son marginales. Estas diferencias pudieran ser explicadas tomando en cuenta queel SP cubre una parte mayor de la población en zonas con una infraestructura de salud muy precaria que las otras instituciones. Otra explicación pudiera ser el subregistro de los servicios prestados pero las diferencias parecen demasiado grandes, particularmente en vista de que parte de la información procede del mismo sistema de información, el SINAIS. 
Tabla 2. Servicios prestados según instituciones públicas, M éxico, 2009.

\begin{tabular}{lcrrr}
\hline \multicolumn{1}{c}{ Tipo atención } & IM SS & ISSSTE & SSA & SP $^{* *}$ \\
\hline Consulta por persona & 3.0 & 3.1 & 3.3 & 1.75 \\
Urgencias por persona & 0.43 & 0.12 & 0.24 & 0.09 \\
Hospitalización por 100 personas & 5.3 & 4.8 & 5.7 & 3.7 \\
Atención Obstétrica \% de la hospitalización & 22 & 15 & 42 & 49 \\
\hline
\end{tabular}

Fuente: *SINAIS9; **CN PSS20 (p. 21-23).

Existen otros elementos que verifican que la afiliación al SP no incrementa el acceso a los servicios requeridos. Un dato importante es el incremento en la mortalidad materna a partir de 2007 a pesar del establecimiento del PES como modalidad del SP. Otra evidencia importantepara el análisises el resultado de la evaluación externa del SP con un diseño cuasi-experimental contratado por la SSA con la Universidad de Harvard. Demuestra que los afiliados al SP no difieren en el uso de los servicios de la población no-afiliada y tampoco respecto al impacto sobre la salud ${ }^{28}$. Esto desmientelos resultados preliminares deeste mismo estudio ${ }^{17}$ que sugirieron un incremento 7 en el acceso y en la cobertura efectiva de 11 intervenciones. Son los resultados preliminares que han sido retomados en varias "evaluaciones" del $\mathrm{SP}$, entreotrospor el Banco Mundial ${ }^{21}$ como prueba del éxito del programa.

Para completar el panorama sobre la posibilidad de que se alcance el aseguramiento universal, algunos datos sobre los seguros privados. En el caso de M éxico los seguros médicos no han crecido como en otros países de América Latina pero los datos sobre ellos son imprecisos. La Asociación M exicana de Seguros sostiene que cubreal 5\% de la población o unos 5.5 millones de personas con algún tipo de seguro de salud, mientras que las encuestas poblacionales, el Conteo de 2005 y el ENIGH de 2008 encuentran un $2 \%$ o poco más de 2 millones de asegurados.

Los seguros privados generalmente se dan como prestación laboral a la alta burocracia, pública y privada, o en la negociación con algunos grandes sindicatos. En ambos casos también son derechohabientes de algún instituto de seguridad social. Hubo cambios importantes en la legislación después de la reforma del IMSS en 1997 para establecer las Instituciones de Seguros Especializadas en Salud (ISES) como administradores privados de los fondos del IM SS. Sin embargo al no prosperar la separación de funciones Ias ISES casi no se han desarrollado.

\section{Conclusiones y una reflexión final}

Es problemático sacar conclusiones sobre el trayecto del Seguro Popular para culminar la reforma mercantilizadora y, por ende, privatizadora desalud en M éxico. Es así porqueel doblediscurso y la ambigüedad en los datos de cobertura y de financiamiento dificultan conocer los hechos reales. Pareceimprobablequesealcancela cobertura universal del aseguramiento tanto por la arbitrariedad en la definición de la población objetivo como por la falta de precisión en las modalidades del SP. Es, sin embargo, probable que se haga una declaratoria de "cobertura universal" a finales de 2011 o inicio de 2012 sin haberlo alcanzado.

Pareceinverosímil conseguir la cobertura universal vía el aseguramiento, particularmentecuando el seguro es voluntario. Habría que recordar que los pioneros del aseguramiento en América Latina-Chile y Colombia-no la han logrado a pesar de que su seguro es obligatorio ${ }^{29}$. No obstante las entidades federativas mexicanas tienen un fuerte incentivo para manipular los datos de afiliación. Son la llave de recursosadicionales, necesarios y políticamente aprovechables, como lo son también para el gobierno central.

No hay que confundir la cobertura poblacional universal con la cobertura médica universal. Este doble significado del vocablo "cobertura universal" ha dado lugar a una considerable vaguedad en el discurso dominante sobre el objetivo de la política de salud. Es notable que los organismos supranacionales la pregonan pero en sus formas más acotadas ${ }^{30,31}$. El SP resulta ilustrativo al respecto ya que el Catálogo Universal deServicios deSalud es muy inferior en su cobertura médica que los seguros médicos de la seguridad social. Incluso se aprecia que la limitada cobertura médica está desl egitimando el SP ante la población. Es además difícil que resuelva sus gastos médicos catastróficos.

A ello hay que añadir la diferencia crucial entre cobertura y acceso. Tener un seguro no debe 
confundirse con tener acceso, ni siquiera, a los servicios amparados en la póliza. El SP lo demuestra. Para los asegurados en los estados pobres donde no existen los recursos humanos y físicos suficientes el seguro es un papel que les autoriza ciertos servicios pero no tienen donde realizar este derecho.

En este contexto conviene alertar sobre las comparaciones de "cobertura" antes y después dela adopción de una política de aseguramiento. El aseguramiento es sólo un mecanismo institucional para acceder a los servicios y no debe confundirse con el acceso real a éstos. Antes de la adopción de la forma "seguro", el acceso o noacceso a losservicios dependía de obstáculos económicos, geográficos, burocráticos o culturales y el aseguramiento en sí sólo remueve una parte acotada del obstáculo económico mientras que los otros obstáculos pueden persistir.

La introducción del modelo de"competencia administrada" básicamente se ha estrellado contra la fortal eza de los institutos públicos de seguridad social a pesar de su prolongado proceso de desgastey desfinanciamiento. En este contexto el SP presiona desde dentro del sector público hacia la separación entre"administración defondos-compra de servicios" y la "venta y prestación deservicios"; separación quees la condición necesaria dela competencia y del mercado en salud junto con la definición deun paqueteespecífico deintervenciones.

El seguro de salud con un paquete predefinido y costeado es conceptualmente distinto al seguro médico de la seguridad social. El primero tiene detrás un concepto de la atención a la persona como un bien privado y la noción del bien público reservada para un número muy restringido de acciones de salud pública. Esto ha tenido un impacto muy importante sobre la salud pública y colectiva quees responsabilidad dela SSA/ SES. La vinculación delosincentivos económicos con el SP ha desplazado las acciones de salud pública al igual que en Colombia ${ }^{32}$. 0 sea, aparte de promover la simulación o franca manipulación de los datos de afiliación el SP tiende a destruir el tejido institucional sobre el cual descansaban la seguridad sanitaria y prácticas de salud colectiva. Por ejemplo, el sistema de vigilancia epidemiológica que se opera desdelos centros de salud y las jurisdicciones sanitarias de la SSA/SES se ha visto afectado tal como se vio en la emergencia dela Influenza A/H $1 N 1^{33}$.

EI SP logra varias cuestiones importantes en la ruta de la mercantilización. Garantiza la capacidad de compra deservicios de los afiliados. O rigi- na esta nueva demanda en ausencia de la infraestructura pública necesaria para satisfacerla, generando un mercado para los servicios privados. Definey costea las intervenciones cubiertas por el seguro, o sea les pone un precio, lo que es indispensable para la compra-venta en el mercado.

Estas características permiten adelantar algunas hipótesis respecto a las inconsistencias encontradas. La errónea clasificación de las familias para el pago de la prima autoriza inscribirlas sin cobro alguno y mantenerlas en el padrón durante tres años. En tanto los subsidios federales no varían en función de la prima del asegurado el fondo de salud para compra de servicios no se alteraría con este procedimiento. Por otra parte, la falta de inversión en infraestructura pública de salud crea, en teoría, un mercado cautivo de venta de servicios privados aunque en la realidad no está probado que los privados recurran a éste. Probablemente ocurrirá en los centros urbanos pero difícilmente en las zonas rurales pobres.

Queda el interrogante sobre por qué se está subfinanciando el SP si se pretendecrear un fondo de salud estable para expandir el sector privado. Habría que analizar este hecho en la dinámica general impuesta al sector público de salud con el suceso del Sistema de Protección Social en Salud. En la nueva lógica y con la presión deestablecer la portabilidad de los seguros entrelas instituciones públicas será necesario definir paquetes de servicios amparados por la seguridad social laboral como ya está previsto en la nueva ley del ISSSTE. Se observa aquí un doble movimiento. Por un lado se compatibilizarían los seguros para hacerlos intercambiables y con ello se abriría un nuevo mercado que es el de los seguros complementarios y/o co-pagos.

La presión sobre los institutos de seguridad social que se crearía con un esquema pleno de portabilidad afectaría todavía más la excedida demanda de servicios por sus derechohabientes y haría aparecer como una solución buena la atención privada. Durante los diez años de gobierno conservador ha estado presente la pugna entre la SSA y el IM SS de la cual la secretaría ha salido ganadora. Se observa en la exigencia de establecer altas reservas actuariales y la falta de inversión en infraestructura, reposición de equipo, contratación depersonal, etcétera. Paralelamente sehaido destruyendo el contrato colectivo delos trabajadores y, con ello, su identidad institucional. Es decir, el proceso que se intentó iniciar a partir dela reforma de 1995 y quese estrelló contra la fortaleza institucional del IM SS avanza hoy en un proceso sistemático de destrucción institu- 
cional. Se aprecia que forma parte dela reorganización de la sociedad con una redefinición profunda y regresiva de cuáles son los "derechos" ciudadanos en salud. En este proceso el Seguro Popular es la palanca.

\section{Referencias}

1. Laurell AC. La política social del pacto posrevolucionario y el viraje neoliberal. En: AC Laurell, coordinador. Hacia una política social alternativa. M éxiCo DF: F Ebert Stiftung e IERD; 1996.

2. Laurell AC. Reforma contra la salud y seguridad social. M éxico DF: ERA; 1997.

3. Laurell AC. Crisis, neoliberal health policy and political processes in M exico. Int J Health Serv 1991; 21(3):457-470.

4. Estatísticas por tema [site na Internet]. [acessado 2010 Abr. 1]. Disponible en: www.sinais.salud.gob. mx/estadisticasportema.html

5. Estadísticas de infraestructura e recursos humanos. [site na Internet]. [acessado 2010 Fev. 15]. Disponible en: www.sinais.salud.gob.mx

6. Amezcua OA. Nueva ley del seguro social comentada. 10a ed. En: Amezcua OA. Sistemas de información contable y administrativa computarizados. M éxiCo, DF: Sicco Gasca; 2002. p. 77-79.

7. World Bank. M exico: Health System Reform [unpublished document]. Washington, DC: World Bank; 1998.

8. Mexico. Instituto Mexicano del Seguro Social (IMSS). Informe al Ejecutivo Federal y al Congreso de la Unión sobre la situación financiera y los riesgos del IM SS: 2006-2007. M éxico, DF: IM SS; 2008. p. 136.

9. Estadísticas de servicios. [site na Internet]. [acessado 2010 Fev. 15]. Disponible en: www.sinais.salud. gob.mx/serviciosotorgados/index.html

10. Ley del Instituto de Seguridad y Servicios Sociales de los Trabajadores del Estado. Diario Oficial de la Federación 2007; 31 marzo.

11. Ulloa O, Raya A. La nueva Ley del ISSSTE. La reforma estructural del consenso dominante. En: Ortiz R, coordinadora. Diez años de reformas de seguridad social en M éxico. México DF: Grupo Parlamentario del PRD Congreso de la Unión; 2007. p. 133-160.

12. México. Instituto Nacional de Estadística y Geografía. Encuesta nacional de ingreso y gasto de los hogares 2008. Aguascalientes: INEGI; 2009. [documento na Internet]. [acessado 2011 abr. 18]. Disponible en: www.inegi.org.mx/inegi/default.aspx? $\mathrm{s}=\mathrm{est} \& \mathrm{c}=10205$
13. M éxico. Comisión Nacional de Protección Social en Salud: Informe de resultados primer semestre 2009. M éxico DF: Secretaría de Salud; 2010.

14. Laurell AC. Health system reform in M exico: a critical review. Int J Health Serv 2007; 37(3):515-535.

15. Molina J. Verificación de la cobertura universal de los servicios de salud. M éxico DF: Organización Panamericana de la Salud; 2002.

16. M éxico. Ley del Seguro Social. Diário O ficial de la Federación 1995; 21 diciembre.

17. Gakidou E, Lozano R, González-Pier E, AbbottKlafter J, Barofsky JT, Bryson-Cahn C, Feehan DM, Lee DK, Hernández-Llamas H, M urray CJ. Assessing the effect of the 2001-06 M exican $\mathrm{H}$ ealth $\mathrm{Re}$ form. Lancet 2006; 368:1932.

18. Decreto por el que se reforma y adiciona la Ley General de Salud. Diario Oficial de la Federación 2003; 15 mayo. [Transitorio 8].

19. Frenk J, González-Pier E, Gómez-Dantés O, Lezana M, Knaul FM. Comprehensive reform to improve health system performance in M exico. Lancet 2006; 368:1524-1534.

20. M éxico. Comisión Nacional de Protección Social en Salud: informe de resultados 2009. M éxico DF: Secretaría de Salud; 2010.

21. Banco M undial. Proyect P116226 Support for the social protection System in Health; 2010, marzo.

22. Dirección General de Información en Salud. Recursosfinancieros, 2000-2008. SICUENTAS; 2010. [documento na Internet]. [acessado $2011 \mathrm{abr}$. 18]. Disponible en: www.sinais.salud.gob. $\mathrm{mx} /$ recursosfinancieros /index.html

23. M éxico. Secretaría de Hacienda y Crédito Público. Informe sobre la situación económica, las finanzas públicas y la deuda pública, cuarto trimestre 2009, anexo XV. México DF: SHCP; 2010. p. 38.

24. Nigenda G. El Seguro Popular de Salud en M éxico. Washington DC: Banco Interamericano de Desarrollo; 2005. [Nota Técnica de Salud No. 2/2005].

25. Auditoría Superior de la Federación. Informe del Resultado de la Revisión y Fiscalización Superior de la Cuenta Pública 2005. En: Auditoría Superior de la Federación. M exico. Informe ejecutivo. M éxico DF: ASF; 2007. p. 179-181. 
26. Sinais de saúde. [documento na Internet]. [acessado 2010 abr. 1]. Disponible en: www.sinais.salud.gob. $\mathrm{mx} /$ estadisticasportema.html

27. Laurell AC, Herrera J. Proyectos de prestación de servicio: una nueva forma de privatización. Médico DF: ALAMES-M éxico; 2010. [Documento de trabajo].

28. King G, Gakidou E, Imai K, Lakin J, Moore RT, Nall C, Ravishankar N, Vargas, Téllez-Rojo MM, Ávila JEH, Ávila M H, Llamas HH. Public policy for the poor? Lancet 2009; 373(9673):1447-1454.

29. Laurell AC, Herrera J. La Segunda Reforma de Salud: aseguramiento y compra-venta de servicios. Salud Colectiva 2010; 6(2):137-148.

30. Gottret P, Schieber G. Health financing revisited. Washington DC: World Bank; 2006.

31. Tsounta E. Lessons for the Eastern Caribbean and beyond: IMF Working Paper. Washington DC: IMF; 2009. [U niversal Health Care, n. 10].

32. Arbelaez MP, Gaviria MB, Franco A, Restrepo R, Hincapie D, Blas E. Tuberculosis control and managed competition in Colombia. Int J Health Plann M gmt 2004; 19(Supl.1):S25-S43.

33. Laurell AC, Herrera J. La influenza A/H 1N 1: análisis del caso mexicano. PoSibles 2010; 5:17-22.

Artigo apresentado em 11/05/2010

Aprovado em 18/10/2010

Versão final apresentada em 18/10/2010 